\title{
BMJ Open Quality Improving monitoring of diabetic complications in home care patients
}

\author{
Wahila Alam, ${ }^{1}$ Shirmila Syamala, ${ }^{1}$ Hanadi Al Hamad, ${ }^{1}$ Sybil George, ${ }^{2}$ \\ Noorudeen Kunnunmal, ${ }^{1}$ Fatma Abdelfattah, ${ }^{2}$ Sunita Chinamma, ${ }^{2}$ Essa Al-Sulaiti ${ }^{2}$
}

To cite: Alam W, Syamala S, Al Hamad $\mathrm{H}$, et al. Improving monitoring of diabetic complications in home care patients.BMJ Open Quality 2017;6:e000053. doi:10.1136/ bmjoq-2017-000053

- Additional material is published online only. To view please visit the journal online (http://dx.doi.org/10.1136/ bmjoq-2017-000053).

Received 14 March 2017 Revised 2 August 2017 Accepted 18 September 2017

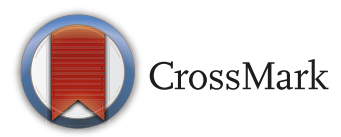

${ }^{1}$ Department of Geriatrics, Hamad Medical Corporation, Doha, Qatar

${ }^{2}$ Home Health Care, Hamad Medical Corporation, Doha, Qatar

Correspondence to Dr Shirmila Syamala, Department of Geriatrics, Hamad Medical Corporation, Doha, Qatar; ssyamala@hamad.qa

\begin{abstract}
Introduction Uncontrolled diabetes mellitus can lead to microvascular and macrovascular complications. Early detection of complications is necessary to prevent endorgan damage and reduce diabetes-related morbidity. In Qatar, the Home Health Care Services of Hamad Medical Corporation caters to about 1000 patients, who solely depend on home healthcare physicians for primary care coordination, which includes management of chronic medical illnesses such as diabetes, stroke, hypertension and anaemia. Due to physician shortage, different physicians new to home care cover patients on different days. This leads to inconsistency of monitoring for many chronic conditions including diabetes and its complications. In this context, we conducted a quality improvement project to improve compliance to monitoring of diabetes complications in Home Healthcare Services by the implementation of a checklist.
\end{abstract}

Methods We initially collected baseline data on monitoring of diabetes complications by chart review. Quality improvement principles and methods were employed to develop a checklist-based intervention to improve screening of diabetes complications by healthcare staff.

Results Following the intervention, checklist completion rate improved from $0 \%-36 \%$ in 3 months to $63 \%$ in 2 years. The healthcare staff's knowledge of monitoring for diabetes complications improved significantly across all monitored parameters. Furthermore, the percentage of patients being monitored for diabetes complications (ie, outcomes) also improved substantially. Monitoring for proteinuria and diabetic retinopathy improved from $10 \%$ and $17 \%$ at baseline to $85 \%$ and $74 \% 2$ years postintervention, respectively.

Conclusion In conclusion, quality improvement methods were successfully used to improve monitoring of diabetes complications according to international guidelines in a very vulnerable population.

\section{BACKGROUND}

Diabetes affects millions of people worldwide. Poor control often leads to microvascular and macrovascular complications such as heart attack, kidney failure, blindness, increased incidence of infections and peripheral arterial disease. This effect is more enhanced in older adults as they have multiple comorbidities and optimal control is often difficult to achieve. Therefore, it is vital to establish standards of care and monitor for these complications to reduce potential harm.
The prevalence of diabetes in adult Qatari population is $16.7 \%^{1}$ compared with a global prevalence of $8.5 \% .^{2}$ Home Healthcare Services (HHCS) of Hamad Medical Corporation (HMC), Doha, Qatar, caters to a population of around 1000 patients. For most of our patients, home care services is the only contact between the patient and healthcare system, with home care physicians filling the gap for primary care providers. HHCS is run through 5 physicians and about 200 nurses. The nursing staff, who provide most of the care, do patient home visits at least once a month. Physicians visit less frequently depending on the condition of the patient.

The older adult population followed by HHCS has an even higher burden of diabetes with more than $50 \%$ of them being affected. We found that only $3 \%$ of these diabetic patients were being monitored for all diabetic complications. International guidelines suggest diabetes monitoring with parameters such as haemoglobin A1c (HbA1c), low-density lipoprotein (LDL), urine microalbumin, creatinine, eye examination, foot examination, weight and blood pressure (BP). ${ }^{3} \mathrm{HHCS}$ physicians were mostly unable to keep track of the parameters to be monitored, due to high volume of patients and paper-based charting system. Inadequate monitoring leads to delay in recognition of complications of diabetes, which leads to increased morbidity. In this context, we designed a checklist for healthcare workers (HCWs) to improve monitoring of diabetes complications in HHCS in Qatar.

\section{METHODS}

\section{Baseline measurement}

We chose one of the HHCS four districts for piloting our project. There were 250 patients in this district out of which 150 patients were known diabetics. We wanted to monitor compliance to the eight parameters suggested by American Diabetes Association for improved diabetes care. All patients who were bed bound were excluded. This was due to logistical issues such as difficulty 


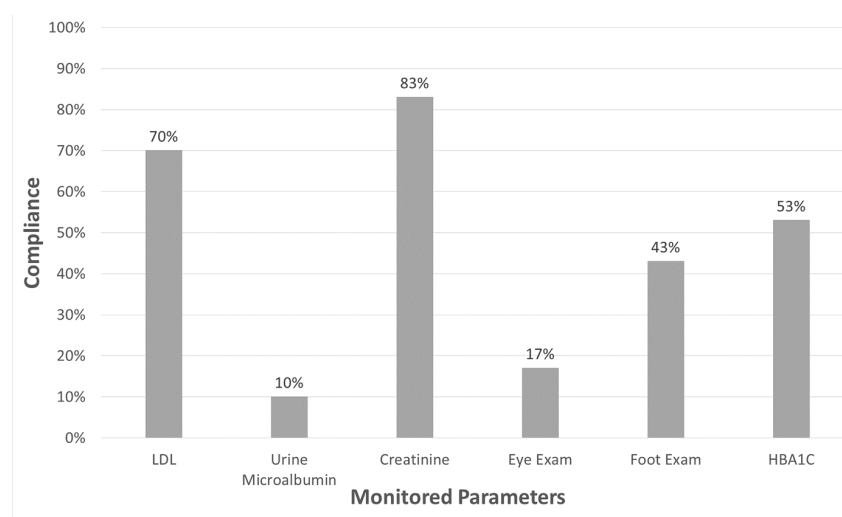

Figure 1 Monitoring for diabetic complications in home care baseline data. HbA1c, haemoglobin A1c; LDL, low-density lipoprotein.

in arranging transportation for an eye clinic visit. Thirty patient files were randomly selected and reviewed from one district for monitoring of HbAlc, LDL, urine microalbumin, creatinine, eye examination, foot examination, weight and BP. Only $3 \%$ of the patients had all eight parameters checked for monitoring of diabetic complications in the last 1 year. Compliance with parameters in testing for microalbuminuria, eye examination, foot examination and $\mathrm{HbAlc}$ were $10 \%, 16 \%, 43 \%$ and $53 \%$, respectively (figure 1 ).

A questionnaire was created to assess the baseline knowledge of the HCWs for monitoring of diabetic patients in home care. It was administered to 40 staff nurses from the same district as well as all 5 physicians in HHCS (figure 2). Out of the 40 staff nurses interviewed, 3\% knew about the foot examination, $5 \%$ about renal function testing, 23\% about yearly cholesterol screening, $30 \%$ about urine protein testing and $46 \%$ about HbAlc monitoring for diabetic patients.

Chart review of the randomly selected patients and Pareto analysis (figure 3) showed that the tests that were most frequently not done were urine protein, eye examination and foot examination. The Pareto analysis showed that urine protein testing and eye examination

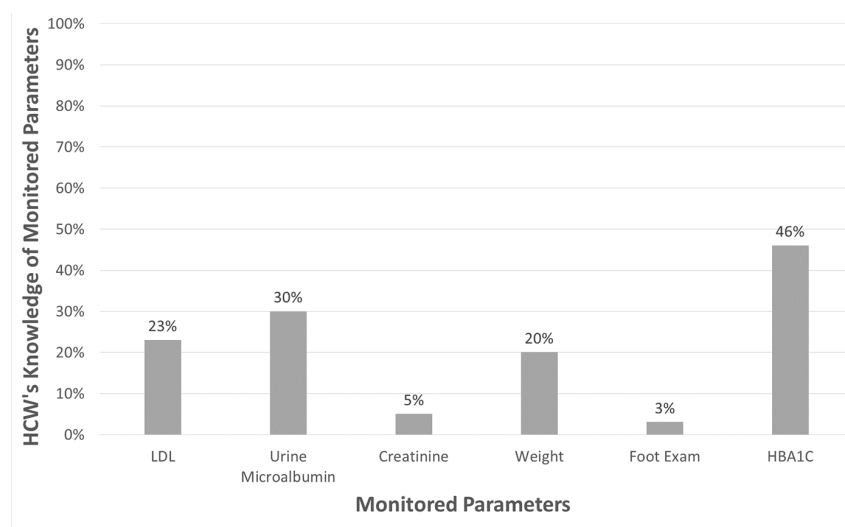

Figure 2 Healthcare workers' knowledge of monitoring of diabetic parameters. HbA1c, haemoglobin A1c; HCW, healthcare worker; LDL, low-density lipoprotein.

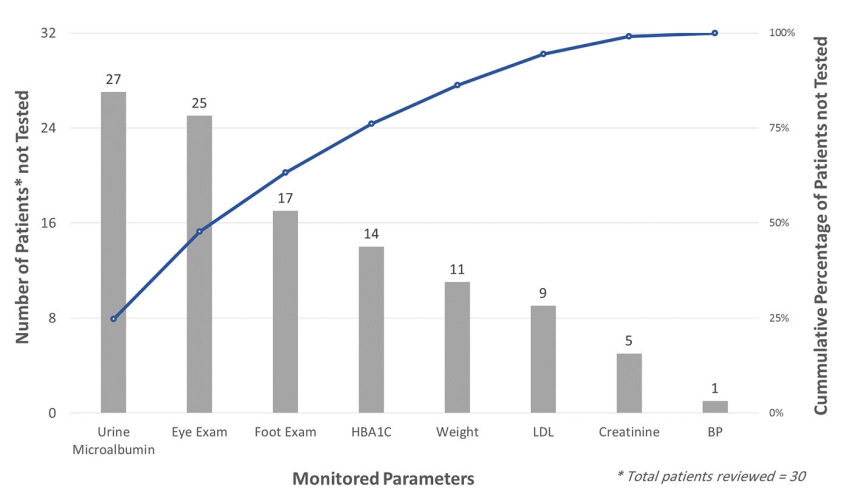

Figure 3 Pareto analysis of monitored diabetic parameters in home care. BP, blood pressure; HbA1c, haemoglobin A1c; LDL, low-density lipoprotein.

alone accounted for more than $75 \%$ of the tests not being done. Although there was some awareness about the need to check for proteinuria, it was not being routinely done. We found that parameters such as weight, LDL cholesterol, creatinine and BP were being monitored more consistently.

\section{Design}

A project team was formed consisting of two physicians, one dietician, one clinical pharmacist, one nurse case manager and one quality reviewer from home care. Checklists for diabetes monitoring were used in various aspects of diabetes care, such as to monitor diabetic wound healing, ${ }^{4}$ improve adherence to diabetes care monitoring guidelines by physicians ${ }^{56}$ and provide guidance for self management. ${ }^{7}$ We planned to explore the idea of using a checklist for optimising diabetes care delivery through home care. A checklist (see online supplementary appendices 1 and 2) was created based on recommended screening guidelines, ${ }^{3}$ which included the following parameters: glycosylated haemoglobin, LDL cholesterol, urine microalbumin, creatinine, eye examination, foot examination and weight. Before using the checklist, many of the parameters were not being ordered and at times missed out completely.

Educational sessions were conducted for all HCWs. These addressed in general the implementation of the checklist and the parameters used in it. Focus was given to the areas where staff knowledge was deficient, such as foot examination, and on tests that were not being implemented despite sufficient knowledge, such as checking for proteinuria.

After the educational sessions, the nursing staff were assigned responsibility for completing the checklist, as they are at the frontline of care. They would review all parameters on the checklist, and if any parameter was not being monitored, they would make sure the appropriate tests and referrals were ordered and followed-up on.

The checklist was then piloted on the 150 diabetic patients in one district. 


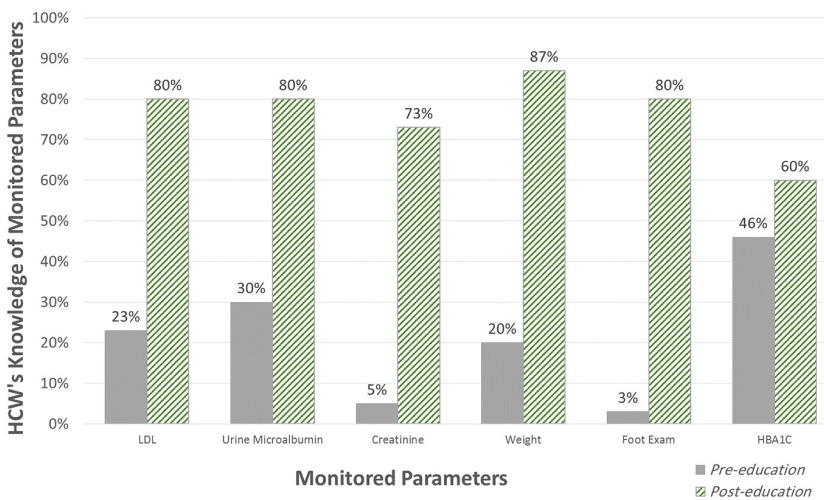

Figure 4 Change in healthcare workers' knowledge after checklist implementation. HbA1c, haemoglobin A1c; HCW, healthcare worker; LDL, low-density lipoprotein.

\section{Strategy}

The initial aim was to increase the percentage of elderly patients monitored for all diabetic complications in Home Healthcare (one district) from $3 \%$ to $30 \%$ by the end of December 2013. The rate of checklist completion was monitored as a process measure. The percentage compliance of monitoring for the six parameters to screen for complications was the outcome measure. Although we attained a checklist completion rate of $30 \%$ by the end of this time, there were still some parameters that were not being monitored.

From January 2014, the project was spread to all districts. Data were collected quarterly to give time to adjust to the new process and changes made.

\section{PDSA cycle 1}

PLAN: By implementing a checklist, diabetic patients will be monitored for parameters such as HBA1c, LDL cholesterol, kidney function, among others, as routine part of diabetes care to prevent complications.

DO: All nurses in the pilot district were educated on the use of the checklist. Nurses, as the primary caregivers, were responsible for implementing the checklist.

STUDY: Files of diabetic patients in three districts were selected randomly $(n=70)$. The checklist completion rate improved from $0 \%$ to $36 \%$ in 3 months.

ACT: As the project seemed to improve diabetes monitoring, the decision was made to implement the change in all four districts. However, despite initial improvement in checklist completion rate, some parameters were not being monitored. Also, there seemed to be a drop in checklist completion rate in later months.

A major reason was felt to be family reluctance and concerns about new tests.

\section{PDSA cycle 2}

PLAN: To improve compliance to the parameters in the checklist that were least addressed.

DO: Feedback was provided about the results of the initial data collection and the tests that were missing. Importance was given to increasing awareness of family members regarding diabetes complications and prevention and the need to do specific tests such as eye examination, foot examination and urine protein testing. Focus would be given to addressing family concerns. Steps were taken to streamline the referral process. We provided ongoing educational sessions to the HCWs.

STUDY: Audit from all districts at the end of second quarter, which showed improved compliance to $68 \%$. However, third and fourth quarter results were again disappointing with compliance of $36 \%$ and $41 \%$, respectively.

ACT: On the basis of the feedback from the multidisciplinary team, we found that a major reason for fall in compliance was due to staff rotation and increased staff turnover. There was a need to establish guidelines as a resource for staff, to ensure sustainability.

\section{PDSA cycle 3}

PLAN: Developing diabetes monitoring guidelines specific to home care will improve compliance with checklist completion. (see online supplementary appendix 3 for Diabetes Guidelines).

DO: On the basis of the international guidelines for similar patients, guidelines were written for home care patients with specific categories including, healthy, complex and very complex diabetic patients. The checklist was revised to include these changes. Nurses were informed on the change in all districts. This took some time, during which there was no data collection.

STUDY: Data collection resumed in fourth quarter of 2015. There was an improvement in monitoring of all parameters, most significantly in the eye examination and urine protein testing. Details are presented in the Results section.

\section{RESULTS}

Follow-up survey to assess the knowledge of HCWs about monitoring in diabetes management showed improved results (figure 4 ). The checklist completion rate (process measure) improved to $36 \%$ soon after implementation of the project and to $63 \%$ 2years later, at the end of 2015. After implementation of the checklist, monitoring of diabetic parameters (outcome measures) improved significantly. Monitoring for proteinuria improved from $10 \%$ to $85 \%$ and for retinopathy increased from $17 \%$ to $74 \%$. There was a very positive trend observed in the monitoring for renal function, glycosylated haemoglobin and annual foot examination, which reached $100 \%$ (figure 5).

\section{DISCUSSION}

In HHCS at HMC, the implementation of a checklist provided a tool for consistent monitoring of diabetes complications and improved the quality of care for patients with diabetes. Before using the checklist, many of the parameters were not being ordered and at times missed out completely. 


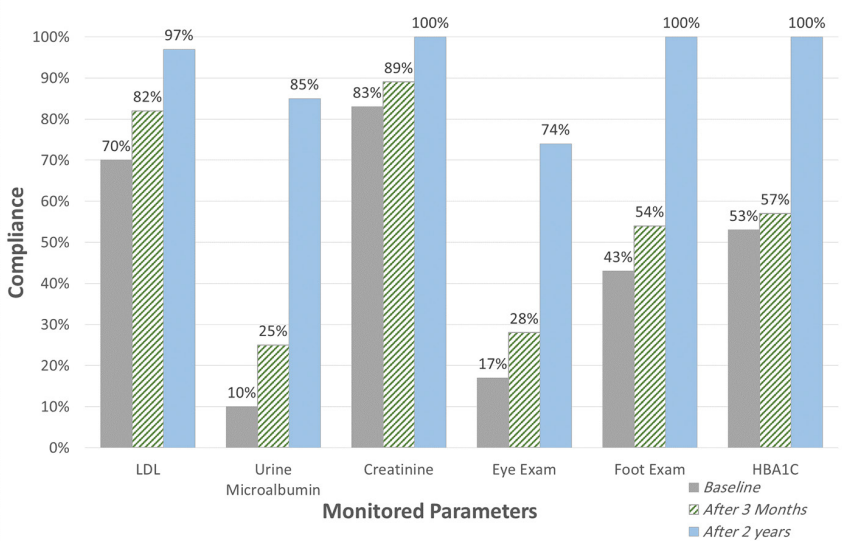

Figure 5 Improved monitoring of diabetic parameters outcome data. HbA1c, haemoglobin A1c; LDL, low-density lipoprotein.

We found that audit of checklist completion and subsequent feedback provided to nurses and physicians helped to improve compliance to diabetes guidelines, consistent with evidence. ${ }^{8}$ Physicians and staff identified the positive impact of the checklist on their ability to care for patients with diabetes. They did not have to rely on memory to order tests/referrals and had a systematic way of documenting the parameters. Continuity of care was ensured, although there was a different HCW assigned. The project also identified the need for the development of clinical guidelines for care of diabetic patients in Home Healthcare. The checklist also made it easier to extract data for quality reviewers to monitor patient care as well as new and rotating staff and physicians to provide consistent care.

The practice of monitoring for development of complications in various chronic diseases has been shown to improve disease management. For example, studies have shown that reminders to curb inappropriate testing for antiepileptic drug levels still resulted in therapeutic drug levels, and using a decision support system for anticoagulation with warfarin led to more patients being within the target range. ${ }^{910}$

Although monitoring is common in clinical practice, the principles of monitoring have not been well conceptualised, which in turn has led to suboptimal care. Chronic care could potentially be improved (and often at reduced costs) if, for each chronic disease, we determined whether and how monitoring was necessary, set explicit monitoring ranges and provided appropriate graphical representations that aided decision making. Health professionals working in chronic care need to understand these principles better and systems need to be improved, including the use of appropriate decision aids. ${ }^{11}$

The choice regarding what to monitor is important as monitoring the wrong indicator can cause unnecessary healthcare utilisation and not yield useful information. For example, in diabetic patients, detecting changes early will impact treatment and with a good monitoring measure will provide an early indication of risk change.
Use of checklists as reminders to HCWs has shown to improve the rate of monitoring for diabetes complications. Use of physician feedback along with an action checklist regarding follow-up, testing and counselling improved adherence to diabetes care guidelines. ${ }^{6}$ Another intervention using a computer-generated checklist for primary care physicians significantly improved monitoring of diabetic complications such as retinopathy, nephropathy and neuropathy. ${ }^{5}$ A world-wide audit of diabetes monitoring using the mnemonic ALPHABET showed inconsistent monitoring for diabetes complications initially, similar to our HHCS. ${ }^{12}$ The same authors then demonstrated that the use of ALPHABET mnemonic as a checklist resulted in better implementation of diabetes care guidelines and improved diabetes care even in resourcepoor situations. ${ }^{13}$

Although we initially faced difficulty with sustainability due to high physician and staff turnover, with consistent project leadership and multi-disciplinary team involvement, this project has been sustained. The checklist completion rate is now considered as one of the process indicators for good-quality care to diabetic patients in HHCS in Qatar. This is followed by quality reviewers in home care on an ongoing basis. The additional cost was not considered a limiting factor due to two reasons: (1) early detection of diabetic complications will prevent morbidity and hospitalisation, thus reducing healthcare costs in the long run, and (2) the state of Qatar provides fully subsidised care to residents, so there is no additional burden to the patients.

This concept of employing a checklist for monitoring chronic disease management is applicable to any patient population followed by modern healthcare teams with roster rotations and staff turnover and will likely help ensure continuity of care over time.

\section{Lessons and limitations}

The biggest lesson learnt was that a small test of change should have been done before implementing the project on a large scale. The checklist could have been implemented on a small number in one district of patients to test the hypothesis. Rolling out the project too quickly in all districts on 600 diabetic patients resulted in waste of resources every time the checklist needed to be changed and waste of time from staff and physicians for education.

One challenge was a drop in compliance with checklist completion by HCWs that we observed after the initial improvement. This could have been due to various reasons. Staff may have felt like they were passive participants in the project. A survey to identify the causes of staff's inability to complete the checklist could have been done. This way, staff may have felt their concerns being heard and encouraged greater participation from their side. The additional paperwork for the HCWs may have been an added burden, but the implementation of electronic medical records in 2015-2016 in Qatar is likely to have reduced this burden. Also, the staff:patient ratio is $1: 20$, so the extra work was found to be feasible 
from HCW feedback as the checklist just needed to be completed only once a year per patient.

Due to logistical reasons, there was insufficient data collection in 2015. However, review in the last quarter of 2015 showed excellent results for monitoring of diabetes complications.

In conclusion, implementing a checklist to monitor diabetic patients improved the quality of care provided to Home Healthcare patients, by improving consistency of monitoring for all chronic complications of diabetes.

Acknowledgements We thank the following: Dana Ahmed, Navas Naddukkandiyil and Sameer Acharath Valappil.

Contributors WA: principal investigator, data collection and manuscript preparation. SS: manuscript preparation and review. HAH: manuscript review. SG: manuscript review, data critique and analysis. NK: data collection. FA: data collection. SC: manuscript review. EAS: manuscript review.

Competing interests None declared.

Ethics approval The work is primarily intended to implement evidenced based care provided to diabetic patients according to the institution's guidelines which do not require ethical approval. We sought only to evaluate the improvements in compliance with the institutions guidelines as a result of auditing and feedback of compliance.

Provenance and peer review Not commissioned; externally peer reviewed.

Open Access This is an Open Access article distributed in accordance with the Creative Commons Attribution Non Commercial (CC BY-NC 4.0) license, which permits others to distribute, remix, adapt, build upon this work non-commercially, and license their derivative works on different terms, provided the original work is properly cited and the use is non-commercial. See: http://creativecommons.org/ licenses/by-nc/4.0/

(C) Published by the BMJ Publishing Group Limited. For permission to use (where not already granted under a licence) please go to http://www.bmj.com/company/ products-services/rights-and-licensing/

\section{REFERENCES}

1 Bener A, Zirie M, Janahi IM, et al. Prevalence of diagnosed and undiagnosed diabetes mellitus and its risk factors in a populationbased study of Qatar. Diabetes Res Clin Pract 2009;84:99-106.

2. World Health Organization. Global report on diabetes. Geneva, 2016.

3. American Diabetes Association. Standards of medical care for patients with diabetes mellitus. Diabetes Care 2003;26(Suppl 1): $: 33-s 50$

4. Martínez-De Jesús FR. A checklist system to score healing progress of diabetic foot ulcers. Int J Low Extrem Wounds 2010;9:74-83.

5. Alfadda AA, Bin-Abdulrahman KA, Saad HA, et al. Effect of an intervention to improve the management of patients with diabetes in primary care practice. Saudi Med J 2011;32:36-40.

6. Schectman JM, Schorling JB, Nadkarni MM, et al. The effect of physician feedback and an action checklist on diabetes care measures. Am J Med Qual 2004;19:207-13.

7. Joslin Diabetes Center. Comprehensive diabetes checklist. http:// www.joslin.org/info/comprehensive_diabetes_checklist.html (2 Jul 2017).

8. Vecchi S, Agabiti N, Mitrova S, et al. Audit and feedback, and continuous quality improvement strategies to improve the quality of care for type 2 diabetes: a systematic review of literature. Epidemiol Prev 2016;40:215-23.

9. Chen P, Tanasijevic MJ, Schoenenberger RA, et al. A computerbased intervention for improving the appropriateness of antiepileptic drug level monitoring. Am J Clin Pathol 2003;119:432-8.

10. Jannuzzi G, Cian $P$, Fattore $C$, et al. A multicenter randomized controlled trial on the clinical impact of therapeutic drug monitoring in patients with newly diagnosed epilepsy. The Italian TDM study group in epilepsy. Epilepsia 2000;41:222-30.

11. Schroeder K, Fahey T, Ebrahim S. How can we improve adherence to blood pressure-lowering medication in ambulatory care? Systematic review of randomized controlled trials. Arch Intern Med 2004;164:722-32.

12. Lee JD, Saravanan P, Varadhan L, et al. Quality of diabetes care worldwide and feasibility of implementation of the Alphabet Strategy: GAIA project (Global Alphabet Strategy Implementation Audit). BMC Health Serv Res 2014;14:467.

13. Lee JD, Saravanan P, Patel V. Alphabet Strategy for diabetes care: a multi-professional, evidence-based, outcome-directed approach to management. World J Diabetes 2015;6:874-9. 